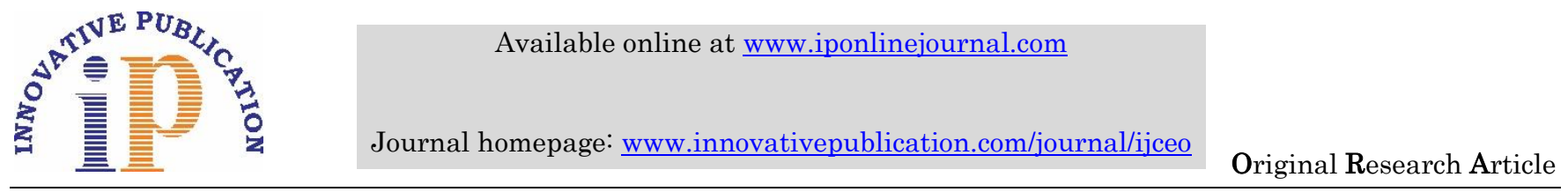

\title{
The study of different sizes and shapes of CCC (Continuous Curvilinear Capsulorhexis) in mature cataracts with \& without trypan blue
}

\author{
Nageshwar Sharma ${ }^{1}$, Rajkumar Raman ${ }^{2 *}$ \\ ${ }^{1}$ Associate Professor, ${ }^{2}$ Junior Resident, Dept. of Ophthalmology, Patna Medical College Hospital, Patna, Bihar, India
}

\section{Article Info}

Received: $21^{\text {st }}$ January, 2019

Accepted: $26^{\text {th }}$ April, 2019

Published Online: $9^{\text {th }}$ September, 2019

Keywords: Capsulotomy, Cataract surgery, Continuous Curvilinear Capsulorhexis / Capsulotomy (CCC), Posterior chamber intra ocular Lens (PCIOL), Trypan blue dye.

\begin{abstract}
The Capsulorhexis or Continuous Curvilinear Capsulotomy (CCC) is perhaps the most important development in the era of modern-day cataract surgery. Continuous Curvilinear Capsulorhexis is one of the vital steps of cataract surgery. Continuous Curvilinear Capsulorhexis of circular shape and about $5 \mathrm{~mm}$ size appears to be the best type of anterior capsulotomy. It helps in long term stable in the bag placement of PCIOL with good fixation and centration with a stretch on the posterior capsule. Very little traction is placed on the supporting zonules during good CCC. The CCC doesn't leave V shaped tear, which have a tendency to extend to the capsular equator or even into the posterior capsular under minimal mechanical stretch and thus renders the capsular bag more resistant to tearing. It has easier cortical aspiration as there is no capsular tags. This stage is best visualized in mature cataracts when stained with Trypan Blue.
\end{abstract}

\section{Introduction}

Cataract is the most common cause of blindness worldwide. So different techniques have been evolved for removal of cataractous lens. The past two decades have seen a rapid development of innovative techniques for improving the safety of Intra Ocular Lens implantation and its postoperative results. One of the most famous surgery is phacoemulsification where the lens nucleus and cortical matter are emulsified by ultrasonic vibration using a hollow $1 \mathrm{~mm}$ titanium needle vibrating 40,000 times/sec, and then removed by simultaneous controlled irrigation and aspiration. The introduction of Phacoemulsification and SICS was due to the need to minimize post-operative astigmatism and day care procedures. There are different method for making window in the anterior Capsule such as Vogt's technique (here the toothed forceps is used for grasping and ripping anteriorly a part of the anterior capsule); Christmas tree approach (here the dull cystitome is used to peel anterior capsule and tore that in triangular or Christmas tree morphology); Can-opener method (here a cystitome for interconnecting perforations of anterior capsule to create a circular window) was found to be unsuitable for Phacoemulsification because the capsular fibre had an annoying tendency to come in the aspiration probe with a high risk of Capsule related complications such as posterior capsular rent and lens drop into the vitreous cavity; Envelope method (or Linear Capsulotomy became increasingly popular in extra capsular cataract extraction as it has advantage of endothelial protection during surgery and surety of 'in the bag' IOL placement. This technique can also be used in hyper mature cataract especially Morgagnian type where there is milky fluid. Here a straight incision is made in the anterior capsule in the upper part from 2 to 10 o'clock position. The rest of the Capsulotomy is completed at the end after removal of cortex and nucleus. But in this case, there is possibility of posterior extension of tear and escape of IOL from the bag and decentration due to long term fibrosis) and CCC. CCC, developed by Howard Gimbel, Thomas Newhann and Schimizu independently in middle 1980s, is the most preferred technique. An anterior capsulotomy opening is made perfectly round without any ragged edges while stained with trypan blue because of easy visualization under microscope. It also permits phaco to be carried out within the capsular bag. The border of capsolorhexis doesn't need to be "circular" but may be ovoid or elliptical in shape. Hence, the generic word curvilinear has replaced the "circular" having the abbreviation the same CCC. The posterior zonular fibres are inserted into 1 to 1.5 $\mathrm{mm}$ posterior to the equator while anterior zonular fibres are attached about $2 \mathrm{~mm}$ anterior to the equator. Since diameter of adult crystalline lens is about $9.5 \mathrm{~mm}$ to $10 \mathrm{~mm}$, the zonular free area on the anterior capsule is approximately $6 \mathrm{~mm}$ in diameter. It is therefore ideal to create a tear limited to the zonular free area preferably slightly the zonular frontier as zonular fibres have occasionally been observed even more centrally in what has been previously considered a zonular free area.

\footnotetext{
*Corresponding Author: Rajkumar Raman, Junior Resident, Dept. of Ophthalmology, Patna Medical College Hospital, Patna, Bihar, India Email: nageshwar_sharma@yahoo.co.in http://doi.org/10.18231/j.ijceo.2019.075
} 
The importance of CCC is very high in case of bag phacoemulsification \& placement of IOL in bag. In mature cataract, there is no fundus glow, so visualization of leading edge of rhexis and getting a planned CCC becomes difficult. A no. of techniques have been advocated \& tried to visualize the advancing edge of the capsule during capsulorhexis. Capsulorhexis can be performed by either a sheer technique or by ripping technique. Both techniques have their own advantages and disadvantages. However, we feel that the sheering technique provides us with an advantage of an ultimate control on the initiation and performance.

\section{Aims}

To study the sizes and shapes of CCC in mature cataracts with \& without trypan blue dye using a bent needle of $23 \mathrm{G}$ to $25 \mathrm{G}$. The first prerequisite is an adequate sized rhexis which should be $5.5-6 \mathrm{~mm}$. When completing the rhexis overlap the tear in such a manner that the last part of the tear joins the first part from the outside towards the center, thus resulting in a continuous edge and making almost circular rhexis in shape.

\section{Materials and Methods}

Random selection of 100 eyes with mature cataracts was done \& CCC was attempted in each of these cases. These cases had been distributed into two groups, gr.1 \& 2. In gr. 1 \& 2 CCC was done with and without using trypan blue respectively. All the cases were done by only one surgeon.

\section{Procedure}

The trend in the cataract surgery has been towards smaller incision moving from superior scleral incision to temporal clear corneal incision, in an attempt to reduce post-operative astigmatism.

For anesthesia and akinesia peribulbar injection is given. After antiseptic dressing with Betadine the bridle suture is placed essentially to maneuver the globe and to fix the same during the various surgical procedures. The bridle suture is placed by grasping the conjunctiva, tenon's fascia and the underlying superior rectus muscle with forceps (approximately 7-10 $\mathrm{mm}$ from the superior limbus at 12 o'clock position). A small fornix based conjunctival flap is made. The conjunctiva is grasped with forceps and a small button-hole incision is made with a sharp tipped scissors along the limbus. The conjunctiva is undermined in both direction and separated with the adherent tenon's capsule from the sclera. A small flap of $8 \mathrm{~mm}$ length and $4 \mathrm{~mm}$ width is often sufficient. Wet thermocautery is applied for hemostasis.

The Scleral tunnel incision has got 3 components: a) External Scleral incision made by 15 number blade B) Sclero Corneal Tunnel is made by crescent knife C) Internal corneal incision into the anterior chamber is made by 2.8 to $3.2 \mathrm{~mm}$ Keratome. The scleral tunnel incision is constructed about $2 \mathrm{~mm}$ away from the limbus, which is astigmatism free zone, perpendicular to the sclera then sclerocorneal pouch is cut followed by oblique entrance incision. The position of incision for side port is 30 degree to 40 degree away from the entry wound. The size of side port is $1 \mathrm{~mm}$ and stab type 15-degree angled knife. It is mainly used for capsulorhexis by $26 \mathrm{G}$ bend needle or for viscoelastic entry.

Through the side port incision anterior chamber is completely filled with air. The air in the anterior chamber causes the dye to spread over the anterior capsule, bordered by the pupillary rim of the iris. It prevents a direct endothelial contact. The air also prevents dilution of the dye by the aqueous. It is observed that a large single air bubble is essential for staining of anterior capsule. Multiple small air bubbles cause irregular staining of the anterior capsule.

After air is injected into the anterior chamber with $27 \mathrm{G}$ cannula, $0.2 \mathrm{ml}$ of $0.1 \%$ Trypan Blue is injected. After 5 to 10 seconds the anterior chamber is thoroughly irrigated with balanced salt solution (BSS) to wash out excess of dye. Because of blue stain of anterior capsule, the outline of the capsulorhexis is clearly visible. This is easily distinguished from the underlying grayish white lenticular tissue thus simplifying capsulorhexis.

Viscoelastic material is injected intracamerally to remove air bubble \& trypan blue. Among viscoelastic substances, Hydroxy Propyl Methyl Cellulose (HPMC) and Sodium Hyaluronate (SH) has become the most popular for intraocular surgeries.

Capsulorhexis is done with 26-G bend needle, because it gives better control of size \&shape of the rhexis. It can also be done with Utarata forceps using main port.

As the rhexis is continued, the control between the stained capsule \& unstained cortex is noted, now following the leading edge becomes easy.

Capsulorhexis is completed.

Hydrodissection, (a fine, 27 gauge blunt-tipped cannula is used to inject BSS under the lens capsule to separate the cortex from the capsule) is then performed. Slight anterior displacement of the cataractous lens is taken as a sign of completion of hydrodissection.

Also, when a slight pressure is given on the ant. Surface of the lens, fluid comes anteriorly from the space between the cortex and posterior capsule of the lens.

Then phacoemulsification process is completed.

I.O.L is placed in the bag.

In the case without trypan blue, the rhexis is done under higher magnification possible and completed slowly \& gently.

There are several advantages of ccc as-there occurs minimal trauma to the zonules, safe and easy aspiration of peripheral cortex, permanent and verifiable fixation of IOL in the bag.

Presence of the visible anterior capsule due to staining with trypan blue allows us to polish posterior capsule.

In case of posterior capsule rupture, it provides good support for sulcus fixation of intraocular lens implant. 


\section{Results}

\section{Rhexis Size}

1. Rhexis size is measured with slit lamp on first postoperative day by relatively comparing with optic size of IOL.

2. In our study $98 \%$ of cases in Gr.1 had complete CCC measuring about $5.5 \mathrm{~mm}$ in diameter. Even though $\mathrm{ccc}$ was obtained in $96 \%$ of gr.2 patients, the size varied from $4.5 \mathrm{~mm}$ to $6.5 \mathrm{~mm}$.

3. Peripheral extensions were noted in $2 \%$ \& $4 \%$ in gr. 1 \& gr.2 respectively.

\section{Table 1}

\begin{tabular}{|c|c|c|c|c|}
\hline Group & \multicolumn{4}{|c|}{ Size } \\
\hline & $4.5 \mathrm{~mm}$ & $5 \mathrm{~mm}$ & $6 \mathrm{~mm}$ & $6.5 \mathrm{~mm}$ \\
\hline Gr.1 & 3 & 22 & 24 & \\
\hline Gr.2 & 19 & 13 & 11 & 5 \\
\hline
\end{tabular}

\section{Rhexis Shape}

We obtained good circular rhexis in majority of cases in gr.1, in gr.2, shapes are so variable \& that few cases required double rhexis to make them circular.

Table 2

\begin{tabular}{|c|c|c|c|c|}
\hline Group & \multicolumn{4}{|c|}{ Shape } \\
\hline & Circular & $\begin{array}{c}\text { Horizontally } \\
\text { Oval }\end{array}$ & $\begin{array}{c}\text { Vertically } \\
\text { Oval }\end{array}$ & Irregular \\
\hline Gr. I & 41 & 0 & 8 & \\
\hline Gr. II & 20 & 12 & 12 & 4 \\
\hline
\end{tabular}

\section{Discussion}

So many techniques have been attempted to improve visualization of ant. Capsule of mature cataracts.

When the density of the cataract does not permit a fundus reflex, controlled capsulorhexis is impaired by reduced visibility of the developing ant. Capsular flap, specially the identification of the leading tear edge. Under these circumstances, it is advisable to significantly increase the microscope magnification or choose additional side illumination as to perform both these maneuvers. It may also prove helpful to manipulate the globe in such a way that reflex of the microscope light on the ant. Capsule remains positioned over the advancing tear edge. Within this specular reflex, the capsular margin becomes clearly visible. Finally choosing a relatively small diameter for the capsulorhexis and proceeding extremely slowly only as actual visualization permits will help to maintain control of the tear.

The difficulty with totally opaque lenses are increased when cataract is intumescent and a hypermature with a totally liquefied cortex, with intumescent cataracts the risk of losing the tear to the periphery is greatly increased through the augmented intra lenticular volume.

In case of hypermature cataracts, the preferred technique is forceps because the necessary counter pressure will not be maintained by the needle for engagement of capsular flap.
Another helpful technique to improve visualization of the form of capsule is oblique illumination. Some operating microscope included oblique light sources that can be used independently of the Co-axial illumination. Alternatively, a fiber optic end illuminator probe can provide oblique illumination to help highlight the capsulotomy edge.

Diathermy-capsulatomy is recently perfected method. Dyes like gentian violet (Gentian violet ((GV) hexamethyl pararosaniline, also known as crystal violet, methyl violet) is a triphenylmethane dye with anti-bacterial, anti-fungal, anti-helminithic, anti-trypanosomal, anti-angiogenic and anti-tumor properties) and indocyanin green (ICG provides enhanced visualization in eyes with opaque cataracts and the absence of red reflex) were tried. Preoperative trypan blue staining of corneal endothelium was studied for elderly 50 years. No unwanted side effects were noted.

In our study, after 6 months of follow up, no intra operative or post-operative complication were noted.

Although, the trypan blue is larger molecule but under normal circumstances does not appear to cross the intact lens zonules. Also the intact anterior hyaloid face would prevent passage of bulk flow of dye into vitreous cavity.

\section{Conclusion}

In Cataract operation proper visualization of anterior capsule after staining with Trypan Blue dye and doing continuous curvilinear capsulorhexis by $26 \mathrm{G}$ bend needle or Utarata forceps, depending upon the case, gives almost a circular or vertical oval anterior capsulotomy having the size of 5.5 to $6 \mathrm{~mm}$ in comparison with the technique where anterior capsule is not stained with Trypan Blue. So, in conclusion, $\mathrm{CCC}$ can be done more precisely and accurately when one uses trypan blue dye in mature cataracts.

\section{Source of Funding: None.}

\section{Conflict of Interest: None.}

\section{References}

1. Melles GRJ, Se Waard, Pameyer JH, Houdiijn Beekhuis W Trypan blue capsule staining to visualize capsulorhexis in cataracts. J Cataracts Refract Surg. 1999;25:7-9.

2. Norn MS. Per operative trypan blue vital staing of corneal endothelium: eight years follow up. Acta Ophthalmol 1980;58:550-5.

3. Pandey SK, Werner L, Escbar-Gomez M, Roigmelo EA, Apple DJ. Dye enhanced cataracts surgery, I. Anyerior capsule staining for capsulorhexis in advanced/white cataracts. $J$ Cataracts Surg. 2000:26:1052-1059

4. Pandey SK, Werner L, Escbar-Gomez M, Roigmelo EA, Apple DJ. Dye enhanced cataracts surgery,iii.Posterior capsule staining to learn posterior continuous curvilinear capsulorhexis. J Cataract Refract Surg. 2000:26:1066-71.

5. Horiguchi M, Miyake K, Ohta I to Y.Staing of the lens capsule for continuous curvilinear capsulorhexisin eyes with white cataract. Archophthalmol 1998;116;535-7.

6. Newsom, T. Hunter T."'indocyanine green staining in traumatic cataracts“. J Cataracts Refract Surg. 2000:26(11):1691-3.

7. Birchall,Wayne: Rayner, Mtthew, K and Tuner, George S.'Inadverdent staining of posterior lens capsulewith trypan 
blue dye during phacoemulsification" Arch Ophthalmol. 2001;119(7):1082-4.

8. Vnlu K. Gentian violet solution for staining anterior capsule" $J$ Cataracts Refract Surg 2000;26(8):1228-32.

9. Eldin, Sheriff A ganal. "Experimental staining of anterior lens capsule in albino rabbits". J Cataract Refracts Surg 25(9):1289-94.
How to cite this article: Sharma N, Raman R. The study of different sizes and shapes of CCC (Continuous Curvilinear Capsulorhexis) in mature cataracts with \& without trypan blue. Indian J Clin Exp Ophthalmol. 2019;5(3):314-7. 